\title{
Analytical model of temperature distribution in metal cutting based on Potential Theory
}

\author{
F. Klocke, M. Brockmann, S. Gierlings, and D. Veselovac \\ Laboratory for Machine Tools and Production Engineering (WZL), Aachen, Germany \\ Correspondence to: M. Brockmann (m.brockmann@wzl.rwth-aachen.de)
}

Received: 05 March 2015 - Revised: 19 June 2015 - Accepted: 26 June 2015 - Published: 10 July 2015

\begin{abstract}
Temperature fields evolving during metal cutting processes have also been of major interest. Temperatures in the tool influence the wear behaviour and hence costs, temperatures in the work-piece are directly responsible for later product quality. Due to the high significance of temperatures, many modelling attempts for temperature fields have been conducted, however failed to deliver satisfying results. The present paper describes a novel analytical model using complex functions based on potential theory. Relevant heat sources in metal cutting as well as changing material constants are considered. The model was validated by an orthogonal cutting process and different real machining processes.
\end{abstract}

\section{Introduction}

Temperatures occurring during metal cutting processes were of major interest for research since scientific investigations in this field are existing. First documented work is accredited to Thompson (1798) who examined the mechanical equivalent of heat when deephole-drilling brass. Even though his objectives for the experimental work were rather aimed at an understanding of the nature of heat itself than understanding of the metal cutting process, his work marked the starting point for consideration of thermal issues in metal cutting. The precise and quantitative determination of temperatures during the metal cutting process in terms of measurements was first conducted from Shore (1925), Gottwein (1925) and Herbert (1926) almost at the same time, who measured temperatures by means of measurement of electromotive forces, i.e. application of thermocouple method. Measurements of heat radiation in terms of pyrometers and infrared camera were first conducted by Schwerd (1933), Ueda et al. (1998) and Müller (2004). Both physical principles have their advantages and disadvantages, however in summary, measurement of temperatures in the metal cutting process are elaborate and mostly error-prone. A recent overview of temperature measurements in material removal processes is given from Davies et al. (2007).

Due to the difficulties in measurement, the need for modelling of temperatures in metal cutting was expressed by re- searchers several times, e.g. Shaw (2005) and Komanduri (2003). Regarding the different types of modelling, i.e. empirical, simulation and analytical, empirical and simulative models mostly fail to be transferable to other circumstances like different cutting parameters, tool and work material and cutting process. This is mainly due to the early linearization of the physical problem. Analytical models, however not applicable on complex cutting engagement situations, strengthen the basic understanding of the nature of temperature distribution and are in principle transferable to all similar physical problems. Due to this reason, the present paper describes the development of a novel approach for analytical modelling of temperatures in the metal cutting process. The derivation and parameterisation are presented in distinct sections of this paper. The model was validated by experimental trials on a fundamental cutting test rig and on a broaching machine. The aim of this paper is to assess the potential of the novel approach presented, i.e. use of the potential theory for prediction of temperature fields in metal cutting.

\section{Analytical temperature models in metal cutting}

In order to derive analytical models for temperature distribution in metal cutting, the governing physical equation, i.e. the partial differential equation for heat conduction has to be 
solved:

$$
\frac{\partial^{2} T(x, y)}{\partial x^{2}}+\frac{\partial^{2} T(x, y)}{\partial y^{2}}=0
$$

The equation is shown for two spatial dimensions $x$ and $y$, stationary and constant heat conductivity, which should be the assumptions for the model derivation in this paper. Physical meaningful solutions can be found either in real-valued functions or complex-valued functions. Nowadays analytical models are exclusively using real-valued functions as will be described in the following.

\subsection{Real-valued functions}

Regarding the various analytical models available, the approach from Komanduri and Hou (2000) can be indicated the most advanced analytical model which was only slightly modified in recent times (e.g. Karas et al., 2013). When analysing their solution, the basic function that was used as a solution for Eq. (1) is one originally invented by Carlslaw and Jaeger (1959) and first applied by Hahn (1951):

$T(x, y)=\frac{q_{l}}{2 \pi \lambda} e^{\frac{-x v}{2 a}} \cdot K_{0}\left(\frac{R v}{2 a}\right)$.

In physical means, the solution can be interpreted as a moving line heat source with velocity $v$. The solution was used by Komanduri and Hou (2000) to form a moving band heat source with an inclination angle relative to the direction of motion. The adiabatic boundaries and further boundary conditions in metal cutting were considered using mirror heat sources, exploiting the resulting symmetrical nature of the temperature distribution plot. Indeed, the vast majority of analytical models are using the Hahn (1951) solution as basic solution and differ from each other only in terms of different boundary conditions and heat source shape, like the model of Carlslaw and Jaeger (1959), which is commonly used in simulation models for temperature prediction.

Most analytical models based on Hahn solution or other similar real-valued functions do not sufficiently model the nature of the metal cutting process or are not validated at all. The main problem occurring is the deviation of the predicted distribution, which suggests that not the right mathematical function describing the nature of metal cutting was found. Furthermore, the existing analytical models are applicable only with restrictions, as the integrals of Eq. (2) are very complex and only symmetrical situations can be modelled when boundary conditions, e.g. adiabatic surfaces or heat sources, are considered.

\subsection{Complex-valued functions}

Besides solutions in the real-valued space, complex functions solving Eq. (1) are existing. A complex function is a function of the complex variable $z$, where $z=x+i y$. The two spatial dimensions are separated by the complex number $i$. The complex function can be expressed as:

$F(z)=\Phi(x, y)+i \cdot \Psi(x, y)$.

The functions $\Phi$ and $\Psi$ itself are real-valued functions and can be plotted in $x$ and $y$ graph. To find solutions of Eq. (1), there is a special group of complex functions called potential functions. These functions fulfil the following requirement:

$\frac{\partial F}{\partial y}=i, \frac{\partial^{2} F}{\partial y^{2}}=\frac{\mathrm{d}^{2} F}{\mathrm{~d} z^{2}} i^{2}$.

Taking into account that $i^{2}=-1$ these functions fulfil Eq. (1) if Eq. (4) is true. If the function $F(z)$ is a potential function then the real part $\Phi$ is orthogonal to the imaginary part $\Psi$ when plotting both in an $x-y$ diagram. For potential functions furthermore the principle of superposition is true, i.e. if $F_{1}(z)$ and $F_{2}(z)$ are solutions of Eq. (1), then also $F_{3}(z)=F_{1}(z)+F_{2}(z)$ is also a solution.

Potential functions are firstly only mathematical solutions of Eq. (1), however they are applied successfully in other engineering fields. Most famous application can be found in fluid mechanics where solutions of potential theory are used to predict stream fields, compare e.g. Anderson (2011). The fact that exactly the same partial differential equation, i.e. Eq. (1), needs to be solved, yields the lack of available solutions for temperature distribution in metal cutting.

\section{Model derivation}

In order to derive new models for prediction of temperature rise distribution for metal cutting, the following method was applied:

- Identification of suitable basic complex functions from potential theory and superposition of these functions.

- Parameter study of each coefficient of the function, assessment of physical relevant numerical range.

- Application of model on metal cutting process, i.e. consideration of relevant heat sources and boundary conditions.

The three steps can be understood with iterative character, especially because the last step, i.e. the application on the metal cutting process is the most important. The results presented in this paper are concluded from a detailed iterative approach using the stated method.

\subsection{Identification of function}

The basic functions used in potential theory can be physically interpreted with the help of the streamline analogy. Figure 1 shows three distinct functions, a uniform flow, vortex and corner flow, when plotting the imaginary function $\Psi$ in an $x-y$ diagram. 


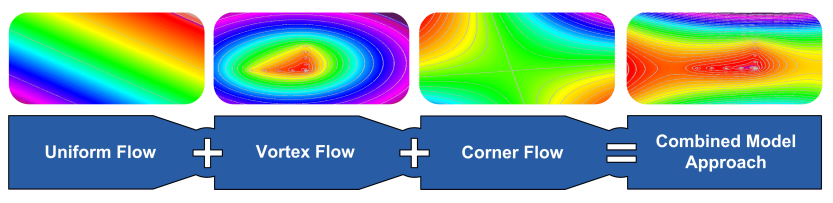

Figure 1. Basic functions and combined modell approach.

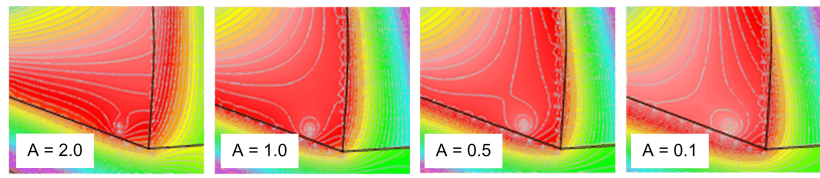

Figure 2. Variation of parameter $A$.

Transferring the physical interpretations to temperature lines for the metal cutting process, detailed analysis of more basic functions revealed that the superposition of these three functions yield a plot which appears similar to the temperature distribution in metal cutting. The not yet parameterised plot of $\Psi$ of the combined function is shown at the right side of Fig. 1. The function $F(z)$ can be expressed as:

$$
\begin{aligned}
F(z) & =F_{\text {uniform }}+F_{\text {vortex }}+F_{\text {corner }} \\
& =A z e^{-i \alpha}+\frac{B i}{2 \pi} \ln \left(z+z_{\text {sum }}\right)+C \cdot z_{\text {rot }}^{k} .
\end{aligned}
$$

The distinct parameters $A, \alpha, B, z_{\text {sum }}, C, z_{\text {rot }}$ and $k$ are described in detail in Sect. 3.2. Taking into account the streamline analogy it appears strange at a first glance that the vortex flow and not a source or sink function is used in the combined function. As stated before this was the outcome of a detailed iterative approach yielding better results in regard to an application on the metal cutting process. The solutions were chosen due to an iterative methodology. Physical interpretations of the solutions, in terms of their influences on the run of the isotherms are known, e.g. the vortex flow bends the isothermal field.

\subsection{Parameter study}

The parameter study of $A, \alpha, B, z_{\text {sum }}, C, z_{\text {rot }}$ and $k$ showed different kind of influences on the temperature plot and hence different kinds of possible interpretations referred to the metal cutting process. Due to the high number of unknown parameters, each parameter was varied taking into account the nature of each parameter for the basic flow analogy, e.g. parameters $A$ and $\alpha$ belong to the uniform flow and influence velocity and angle of the flow. Figure 2 shows the variation of parameter $A$ as an example. The parameter study shows that a variation of $A$ from 0.1 to 1.0 does not change the shape of the temperature distribution field significantly, the isotherms shape does vary with rising value of $A$ but the general behaviour stays constant.

The plot in the upper left of Fig. 2 in contrast, shows a behaviour which is not typical for temperature distribution in
Table 1. Numerical values for $F(z)$.

\begin{tabular}{lrl}
\hline Parameter & Values & correlation to ... \\
\hline$A$ & $0.1-1.0$ & heat source strength \\
$\alpha$ & $0.5-1.2$ & cutting speed, chip thickness \\
$B$ & $11.0-14.0$ & cutting speed \\
$C$ & $0.20-0.26$ & chip thickness \\
$k$ & 1.85 & chip thickness \\
$\theta$ & $(0.025-0.05) \pi$ & shear zone angle \\
\hline
\end{tabular}

metal cutting, i.e. the isotherms run into the rake face of the tool, which is a discrepancy to the known literature results and own experimental trials that are presented later in this paper. In a similar way of parameter study, numerical sensible values for each parameter were determined, summarised in Table 1.

The last row of the table gives an indication of possible correlation to cutting parameters and nature of the heat sources shear and friction. However these values are only yielded by observation and were not validated. The major conclusion of the parameter study shows that all shown parameters stay in a relative narrow numerical band when considering that apart from any experimental data only extreme situations were used to define the numerical boundaries as described in Fig. 3. In Table 1, the parameter $\theta$ is replacing the term $z_{\text {rot }}$ from Eq. (5) considering:

$z_{\text {rot }}=\sqrt{(x \cos \theta-y \sin \theta)^{2}+(x \sin \theta+y \cos \theta)^{2}}$.

Summarised, the parameters presented in this Section seem to be almost constant respectively only slightly changing for all temperature fields that can be predicted with this model approach. The parameter $z_{\text {sum }}$ in Eq. (5) however was found not to be constant.

\subsection{Application on metal cutting process}

Considering the stream flow analogy of Eq. (5), the term $z_{\text {sum }}$ describes number and direction of vortexes that can be placed at different locations. The term $z_{\text {sum }}$ can be expressed as:

$z_{\mathrm{sum}}=z_{\mathrm{ch}}+z_{\mathrm{sh}}+z_{\mathrm{con}}$,

where the term $z_{\mathrm{ch}}$ locates the vortexes on the contact line between rake face and chip, the term $z_{\text {sh }}$ considers the strength shear plane heat source and $z_{\text {con }}$ the length and angle of the shear plane, compare Fig. 3.

For term $z_{\text {ch }}$ the expression

$z_{\mathrm{ch}}=\sum_{-1}^{m}\left(x_{\mathrm{ch}, m}+i \cdot y_{\mathrm{ch}, m}\right)=\sum_{-1}^{m}[1+i \cdot(2 m+1)]$

can be formulated. The number of vortexes along chip side hereby is $m$. The initial negative value under the summation is due to the chosen coordinate system. For the vortexes 

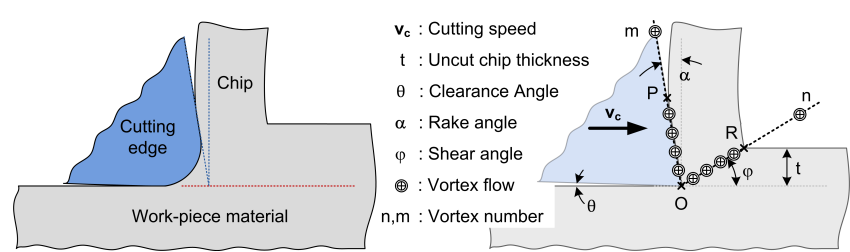

Figure 3. Vortex locations and metal cutting process.

along the shear plane heat source pertains:

$$
\begin{aligned}
z_{\mathrm{sh}} & =\sum_{0}^{n} x_{\mathrm{sh}, n} \\
& +i \cdot y_{\mathrm{sh}, n}=\sum_{0}^{n}\{(2 n+1)+i \cdot[(2 n+1) \cdot \tan (\phi)]\}
\end{aligned}
$$

where $n$ is the number of vortexes along the shear plane and $\varphi$ is the shear angle. The last term $z_{\text {con }}$ can be formulated as:

$z_{\mathrm{con}}=t+i \cdot 0.5 \cdot \mathrm{OR} \cdot \cos (\phi)$

where $t$ is the uncut chip thickness, OR is the length of the shear plane heat source and $\varphi$ again the shear angle. Plugging Eqs. (8), (9) and (10) into Eq. (5) yields the following expression for the function $\Psi(x, y)$ :

$\Psi_{\text {total }}(x, y)=A \sin (\alpha)-\frac{B}{2 \pi} \ln \left(\sqrt{x_{\text {total }}^{2}+y_{\text {total }}^{2}}\right)+C$
$\cdot\left[\sqrt{(x \cos \theta-y \sin \theta)^{2}+(x \sin \theta+y \cos \theta)^{2}}\right]^{k} \sin (k \theta)$.

As stated in Sect. 2.2, $\Psi$ hereby is only the imaginary part of $F(z)$, i.e. all expressions that contain the imaginary unit $i$. The real part of $F(z)$ is not plotted but can be considered as a graph with orthogonal lines. The terms $x_{\text {total }}$ and $y_{\text {total }}$ are defined as:

$$
\begin{aligned}
x_{\text {total }} & =x+1+t+\sum_{0}^{n}(2 n+1) \\
y_{\text {total }} & =y+0.5 \cdot \mathrm{OR} \cdot \cos (\phi) \\
& +\sum_{0}^{n}(2 n+1) \tan (\phi)+\sum_{-1}^{m}(2 m+1) .
\end{aligned}
$$

Plotting the function $\Psi(x, y)$ yields a graph which need to be scaled to get temperature distribution fields, where the parameters $A, \alpha, B, C, \theta$ and $k$ can be considered as nearly constant as described in Sect. 3.2. The uncut chip thickness $t$ is known, solely length of the shear plane heat source OR and number of vortexes on shear plane $n$ and friction zone $m$ have to be measured or modelled.

\section{Model validation}

The presented model in form of Eqs. (11) and (12) were parameterised using the findings from the parameter study.
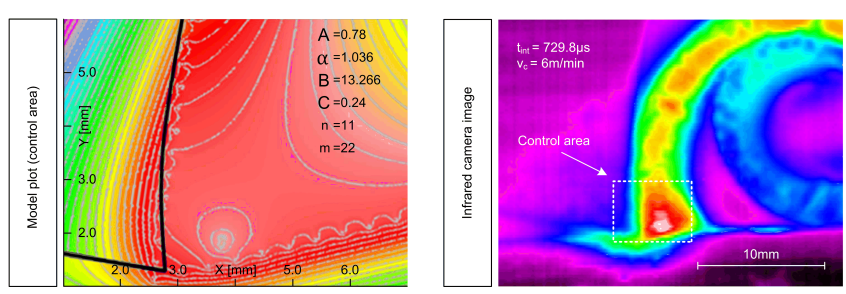

Figure 4. Comparison of model plot and infrared image (for Inconel 718, HSS tool, cutting depth $30 \mu \mathrm{m}$ ).

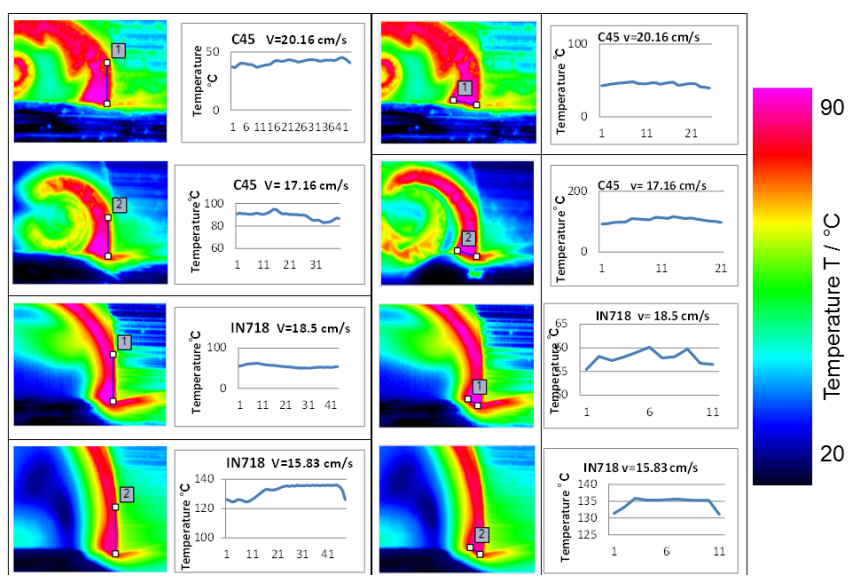

Figure 5. Model validation for Inconel 718 and C45 steel.

The mentioned data for length of shear plane heat source and shear angle were taken out of an infrared image for simplification reasons. Figure 4 shows a comparison between the parameterised model and results from experimental data. As only the shape of the isotherms is of importance no scale is provided.

The experiments were conducted on an analogy test rig for orthogonal metal cutting, the test rig is described in Klocke et al. (2011). The infrared images were taken by an online calibration measurement method combining two-colour parameter with a high-speed infrared camera, details can be found in Gierlings and Brockmann (2013).

The comparison shown in Fig. 4 shows a good accordance in the chip and near the contact zone between tool and work piece, especially in terms of temperature field distribution. The line in the model plot shows that the tool geometry can be found in the model plot. The comparison presented here should be understood as a first qualitative validation showing promising results for the potential of the derived model.

Further validation work was done for a broaching process which can also be found in Gierlings and Brockmann (2013). Figure 5 shows further validation trials for steel $\mathrm{C} 45$ and an Nickel-based alloy (Inconel 718).

The temperature rise distribution along the shear plane heat source and frictional heat source for chip-tool interface was found by using experimental result for steel material C45 and IN718 respectively at different cutting speeds. Therefore, 
the average temperature for these examples was considered to be a reference for calculate the temperature between the shear plane heat source and frictional heat for chip-tool interface. Figure 5 shows the temperature distribution for the $\mathrm{C} 45$ and IN718 work material along the shear plane heat source for different cutting velocity with plots the temperature along the selected profile length. The following parameters were used from Shaw (2005):

- Chip velocity: $v_{\mathrm{ch}}=60 \mathrm{~cm} \mathrm{~s}^{-1}$

- Friction force: $F_{\mathrm{f}}=605 \mathrm{~N}$

- Heat flux: $q=605 \times 60 / 100 /(0.0833 \times 0.025)=$ $68042.8 \mathrm{~W} \mathrm{~cm}^{-2}{ }^{\circ} \mathrm{C}$

- Chip thickness: $t_{2}=0.0833 \mathrm{~cm}$

- Shear angle: $\varnothing=16.7^{\circ}$

The chosen model parameters are:

- Number of vortex flow along the shear plane line: $\mathrm{OR}=11$

- Number of vortex flow along the chip-tool interface line: $\mathrm{OP}=22$

- Parameter for uniform flow: $A=D \times\left(q^{\prime \prime}\right) / / \lambda=5 \times$ $10^{-5} \times 68042.8 / 0.436=0.78{ }^{\circ} \mathrm{C} \mathrm{cm}^{-1}$

- Parameter of (Alpha): $\alpha=v_{c} t_{1} /(40 \times a)=1.036$

- Parameter for vortex flow: $B=E \times v_{c} / a=8 \times 10^{-3} \times$ $200 / 0.1206=13.266 \mathrm{~cm}^{-1}$

- Parameter for corner flow: $C=r /(5.5 w)=0.24 \mathrm{~cm}^{-1}$

- Parameter for corner flow: $k=1.8, \theta=\pi / 30$.

The temperature rise along the chip-tool interface is also calculated. The average temperature rise distribution are considered, this will be also the reference temperature that can used to approximate the temperature rise in the chip-tool interface for the complex potential flow model. Using the average value for temperature rise distribution for the shear plane heat source and chip-tool interface, the combined effect for both the shear plane heat source and chip-tool interface frictional heat source can be represented by using this data. For the final plots for the temperature rise distribution further calibration was required.

\section{Summary and discussion}

The presented paper shows a novel approach for analytical modelling of temperature field distribution for metal cutting processes. For the derivation of the model, complex functions solving the partial differential equation for heat conduction were considered. The complex functions belong to a certain group of functions called potential functions, which already showed good results in other engineering fields e.g. fluid mechanics, where solutions of exactly the same partial differential equation are needed. The analysis revealed the combination of three basic functions to yield temperature plots that show a huge potential for application in the metal cutting process. The combined function was parameterised to suit the situation in metal cutting, mainly by taking into account length and strength of the two heat sources friction and shear zone. Beyond this first approach of using complex functions of the potential theory for modelling temperature fields in metal cutting, still a lot of parameters need to be correlated systematically to the cutting parameters. The final verification of the model is outstanding as more cutting process need to be investigated using the basic function described in this paper.

Acknowledgements. The presented modelling work is part of project A02 of the Collaborative Research Center SFB/TR 96: "Thermo-energetic design of machine tools", funded by the German Science Foundation DFG.

Edited by: M. Cotterell

Reviewed by: G. Fromentin and one anonymous referee

\section{References}

Anderson, J. D.: Introduction to flight, McGraw-Hill Science, 7, 251-362, 2011.

Carslaw, H. S. and Jaeger, J. C.: Conduction of heat in solids. 2nd ed. Oxford [Oxfordshire], New York, Clarendon Press, Oxford University Press, 1959.

Davies, M. A., Ueda, R., M'Saoubi, R., Mullany, B., and Cooke, A. L.: On the Measurements of Temperature in Material Removal Processes, CIRP Annals Manufacturing Technology, 56, 581604, 2007.

Gierlings, S. and Brockmann, M.: Analytical Modelling of Temperature Distribution using Potential Theory by Reference to Broaching of Nickel-Based Alloys, Adv. Mat. Res., 769, 139146, 2013.

Gottwein, K.: Die Messung der Schneidentemperatur beim Drehen, Maschinenbau Betrieb, 4, 1129-1135, 1925.

Hahn, R. S.: On the temperature development at the shear plane in the metal cutting process, Proc. of First National Congress of Applied Mech., 661-666, 1951.

Herbert, E. G.: The measurement of cutting temperatures, Proceeding of the Institution of Mechanical Engineers, 1, 289-329, 1926.

Karas, A., Bouzit, M., and Belarbi, M.: Development of a thermal model in the metal cutting process for prediction of temperature distributions at the tool-chip-workpiece interface, J. Theor. Appl. Mech., 51, 553-567, 2013.

Klocke, F., Bergs, T., Busch, M., Rohde, L., Witty, M., and Cabral, G. F.: Integrated approach for a knowledge-based process layout for simultaneous 5-Axis milling of advanced materials, Adv. in Tribology, 2, 108-115, 2011.

Komanduri, R.: NSF workshop on research needs in thermal aspects of material removal, Oklahoma, 2003. 
Komanduri, R. and Hou, Z. B.: Thermal modeling of the metal cutting process - part I, Int. J. Mech. Sci., 42, 1715-1752, 2000.

Müller, B., Renz, U., Hoppe, S., and Klocke, F.: Radiation thermometry at high-speed turning process, J. Manuf. Sci. E.-T. ASME, 126, 488-495, 2004.

Schwerd, F.: Über die Bestimmung des Temperaturfeldes beim Spanablauf, Zeitschrift des VDI, 9-77, 211-216, 1933.

Shaw, M. C.: Metal cutting principles, Oxford University Press, 2, 29-30, 2005
Shore, H.: Thermoelectric measurement of cutting tool temperatures, J. Washington Academy of Science, 15, p. 85, 1925.

Thompson, B.: An inquiry concerning the source of heat which is excited by friction, Philos. T. R. Soc. Lond., 18, 278-287, 1798.

Ueda, T., Sato, M., and Nakayama, K.: The temperature of a single crystal diamond tool in turning, Annals of the CIRP, 47, 41-44, 1998. 